\title{
Degree of knowledge of root resorption of legal representatives in patients treated in the area of dentistry
}

\begin{abstract}
This literature review is based on the degree of knowledge of root resorption of legal representatives in patients who are treated in the area of orthodontics at the dental clinic of the Catholic University of Cuenca. Knowing the importance of orthopedic treatment, as throughout this process can have different complications such as sterile inflammation of the periodontal ligaments, which produce long term root resorption. This can be caused by several factors such as excessive force at the time of treatment, equipment used, and the treatment time. It is therefore important to have the right skills, before making and the child undergo treatment, so we can prevent future complications.
\end{abstract}

General purpose: Determine the degree of knowledge of the legal representatives of children treated orthopedic through surveys of the same, in order to obtain information about their knowledge.

Materials and methods: The following research work will be conducted using scientific papers obtained at: S-cielo, Pub-med, Google-Scholar, and D-space, which have been made in the last 10years, plus a survey record was created which met criteria for basic diagnostics to assess the degree of knowledge of root resorption in the legal representatives of children treated orthopedically, this tab was valued by an endodontist academic couple, the same that was filled by the investigator interrogating the investigation.

Conclusion: It was concluded that most parents do not know the difference orthopedics and orthodontics but equally take their children to orthopedic treatment because of the limited information they have, it comes to removing the dental crowding of the teeth without knowing much part of the treatment. But they have positive knowledge of the elements of oral hygiene but do not know how to perform dental hygiene properly due to lack of information professionals traffickers. It could also conclude that most of the parents of these children know and are aware that a good dental and orthopedic treatment depends on frequent views dentist to correct their dental condition and give a good occlusion and esthetics child patient. In the same way delve who do not know whether the treatment brings consequences if prolonged long but equally attend the faculty of the University because they want to prevent a crowded teeth future of their children and thus get dental harmony.

Keywords: orthodontic treatment, root movement, Bone resorption, tooth movement
Volume 9 Issue 5 - 2018

\author{
Ronald Ramos Roossevelt Montiel,' Daniel \\ Emanuel Ramírez Romero, ${ }^{2}$ Felipe Rafael \\ Calle Jara, ${ }^{3}$ Carlos Roberto Naula Vicuña, ${ }^{4}$ \\ Miriam Fernanda Ortega López, ${ }^{5}$ Nube \\ Valeria Auquilla Pulgarin ${ }^{6}$ \\ 'General Dentist, Higher Diploma in Integral Dentistry, \\ Specialist in Orthodontics, PhD Student University of Zulia \\ Maracaibo Venezuela, Postgraduate Professor of the University \\ of Guayaquil, Professor of Undergraduate Catholic University of \\ Cuenca, Coordinator of Specialization in Orthodontics Matrix \\ Cuenca, UCACUE, Ecuador \\ ${ }^{2}$ General Dentist, Specialist in Orthodontics, Professor of \\ Undergraduate Catholic University of Cuenca, Coordinator of \\ Specialization in Orthodontics, Azogues extension, UCACUE, \\ Ecuador \\ ${ }^{3}$ General Dentist, Specialist in Implantology Buco Maxilo Facial, \\ Professor of Undergraduate Catholic University of Cuenca, \\ Ecuador \\ ${ }^{4}$ General Dentist, Specialist in Periodontics and Implantology, \\ PhD Student University of Zulia Maracaibo Venezuela, Professor \\ of Undergraduate Catholic University of Cuenca, Ecuador \\ ${ }^{5,6}$ General Dentist, Catholic University of Cuenca, Ecuador
}

Correspondence: Ronald R Ramos Montiel, General Dentist, Higher Diploma in Integral Dentistry, Specialist in Orthodontics, PhD Student University of Zulia Maracaibo Venezuela, Postgraduate Professor of the University of Guayaquil, Professor of Undergraduate Catholic University of Cuenca, Coordinator of Specialization in Orthodontics Matrix Cuenca, UCACUE, Ecuador,Tel+593 0983528857, Email ronald_mtz@hotmail.com

Received: August 06, 2018 | Published: October 29, 2018

\section{Root reabsorption definition}

Root resorption in teeth treated with brace is due to poor treatment by the treating professional to the case, thereby producing mechanical pressure in the periodontal ligament. This path causes damage to tissue of the tooth root due to the loss of the anchorage of the tooth. ${ }^{1}$ Root resorption will result when the pressure in the area of cement is greater than the bearing capacity, affect the repair and exposing the dentin to release multinucleated odontoclasts separating them from their Root. ${ }^{1}$

\section{Root resorption "Etiology"}

The etiology of root resorption has two stages: a stimulus and rechallenge. In the first stage, mineralized tissue is invaded by multinucleated cells which are responsible for the resorption radicular. ${ }^{2,3}$ To give root resorption osteoblasts odontoclasts and osteoclasts involved, and together with non-elastic mononuclear cells. ${ }^{3}$ In the second stage the process of resorption is stimulated by the pressure odontoclastic cells. Multinucleated cells resorb some areas of cementum, by removal of necrotic tissue with macrophage cells, act in removing necrotic tissue and reabsorb some areas of cementum. It should remove all damaged to cease the apical resorption and start the repair process cementogénesis tissue. ${ }^{2}$ Several authors concluded that by eliminating excessive forces root resorption is suspended. However another study investigates, that after treatment there root resorption, but can eliminate the cause when all necrótico tissue is removed. ${ }^{2}$ 


\section{Functional features therapy orthopedic funcional}

Orthopedics is relevant in almost most cases in children patients who are in growth stage. Boys have more advantage than girls because of their skeletal growth is a bit late, so they can be treated. ${ }^{4}$ A very important factor is the behavior of the tongue neuromuscular therefore the balance of the maxillary and mandibular arches of teeth is determined by the relationship between the perioral musculature and the tongue. Orthopedic appliance is helpful because it allows direct the growth of skeletal structures, thus causing, tissue changes, and correcting the dysfunction developed as a result of biodynamic equilibrium. ${ }^{4}$ The objective of this appliance is to avoid oral malocclusions in ther future of the child, and thus to provide a stable or ideal occlusion. ${ }^{4}$

\section{Treatments used in orthopedics}

These treatments are carried out by the existence of devices that can be fixed or removable, all with the same objetive of performing preventive treatments. ${ }^{4}$

\section{Characteristics of removable functional apparatology}

i. They are large appliances.

ii. You may have an unstable retention

iii. Cause discomfort.

iv. Discharge pressure on the supporting tissues.

v. Decreases the space of language.

vi. You can alter the appearance facial. ${ }^{4}$

$\begin{array}{ll}\text { Grade } 0 & \text { There will be no root repsorption } \\ \text { Grade I } & \text { There is a small is repsorption considered mild } \\ \text { Grade 2 } & \begin{array}{l}\text { Presence of small reabsorption considered moderate } \\ \text { 3rd }\end{array} \\ \text { Grade } & \begin{array}{l}\text { The loss of nearly a third of the root resorption accentuated } \\ \text { considered evidence }\end{array} \\ \text { Grade } 4 & \begin{array}{l}\text { Presence reabsorption loss over a third length, considered } \\ \text { extreme reabsorption }\end{array}\end{array}$

\section{Features dental braces}

i. They do not rely $100 \%$ on patient cooperation.

ii. They are designed for your activity during 24 hours.'

iii. There is no difficulty in phonation, swallowing and breathing.

iv. The forces applied these devices allow any modification in the basal bone, and in the same say in the dental pieces that are subject to it. $^{4}$

\section{Frequency root reabsorption}

The most affected teeth on the root resorption stage Interoceptive and corrective orthodontic treatment, are permanent parts $90.5 \%$, In the upper arch or teeth involved is most affected: the upper part 1.1 and 2.1, continuing molars. In the mandibular arch affect central incisors and lateral incisors. The measure of resorption that loses these pieces is approximately $2 \mathrm{~mm}$ in length at the level od the root apex.

A recent study of a group of teenagers, has been shown that root resorption is more severe in them, with root resorption greater than $3 \mathrm{~mm} .^{5}$

\section{Classification of root resorption}

We have different criteria of root resorption and frequently used are:

i. Depending the location: We can find 2 types of resorption, internal and external affecting the root apex.

ii. Depending on their nature: It may be of inflammatory origin.

Root repair can be treated when the force factors are eliminated, in this way it is repaired physiologically by the presence of secondary cement. Histologically studies evidenced that there is a high prevalence of root resorption by orthopedic or ortodontic treatment. Another more widely used classification is being analyzed by degrees depending on the root resorption, these can be evidenced by using panoramic and periapical radiographs as clinical eye can not be observed, therefore, it is necessary to further studies. It has been classified by degrees depending on the reabsorption of the tooth root and we have:

\section{Risk factors for root resorption}

\section{Risk factors etiology pathogenic}

There is a close relationship with systemic and local factors of the individual. In order to prevent its occurrence and monitor their development it is necessary to know the etiological factors and take appropriate measures to prevention and treatment attitudes. We can classify the risk factors into two large groups.

i. inborn
ii. procured

\section{Risk factors inborn}

\section{Individual hereditary predisposition}

Heredity has not been demonstrated today that is a factor transmisible.

\section{Sex}

More frequent studies showed that occurs most often in women than men.

\section{Individual susceptibility}

The susceptibility of the person is different from others, you should make frequent radiographic studies to see if the patient has severe or mild resorption.

\section{Dentoalveolar structure}

It is important that the roots are located in healthy bone pathologies as if this tends to create damage periodonto level thus producing root resorption, with the consequent drop in the same.

\section{Tooth morphology and size}

The teeth which have thin roots and thin dentinal tubules are prone to this disease. 


\section{Tooth type}

The most frequently affected parts will be 1.2 , followed in the limelight with 2.1 parts; $1.1 ; 31 ; 41$, and continues with the mandibular first molar and, finally the second premolar. The upper incisors are more susceptible because its root is tapered and migrate more easily and they present a thin cortical which facilitates movements.

\section{Genetics}

Genetic causes are to play an important role in root resorption present with the application of tension within the orthodontic treatment. It is recognized that some patients are more susceptible than others to present condition, and sometimes this trend is observed in several members of the same family.

\section{Race}

There are more predisposed in whites and Hispanics in Asian.

\section{Age}

The presence of root resorption increases with age and is more susceptible incisors, those parts which are subjected to large movements and when presented atypical root morphology before treatment.

\section{Acquired factors}

\section{RRIO (Root Reborriation induced by Orthodontics)}

It is orthodontically induced inflammatory root resorption, to be produced clinically excessive dental mobility, RRIO is related to several factors:

\section{Genetic}

Recent studies claim that RRIO, can be caused by genetic factors, for that reason it is recommended that clinical and radiographic examination, ah family members who have undergone orthodontic treatment to check the degree of integrity of race.

\section{Race}

Studies have shown that Hispanic race is more prevalent to suffer RRIO that Asian race.

\section{Sex}

Studies have concluded that the type of genre, is not a major factor because in people 25 to 45 years have the same effects of RRIO.

\section{Age}

Root resorption will always increase with age, which is attributed to pathological changes that will suffer the years the periodontal ligament and occlusal change in teenagers patients.

\section{Habits}

Habits before starting orthodontic treatment is one of the most committed factors, and to increase the degree of RRIO, either before, during or after treatment. Among the most common habits it is: onicofagia, bruxism, tongue thrust, dysfunctions lip, lip interposition and middle finger sucking at 7 years.

\section{Systemic factors}

More often inflammatory mediators have presented asthmatics, so will suffer a greater degree of RRIO, affecting more often teething posterior.

\section{Epidemiology}

Root resorption is a phenomenon with a high frequency in orthodontic treatments, which especially occurs in the central incisors, several studies have shown that $90 \%$ of treatments will tolerate root resorption.

\section{Diagnosis}

The most effective way to diagnose RRIO, so is based on panoramic radiography, Rx. periapical, cephalometric and cone beam computed tomography. I recommend an x-ray is taken every three months for radiographic studies and thus acquire records updated data about the dental movement.

\section{Forecast}

During orthodontic treatment effects as root resorption that is 1 to $2 \mathrm{~mm}$ in the upper incisors, which is acceptable to the $3 \mathrm{~mm} .1-2$ Prognosis crown-root is derived from the relationship presented 1a1. The teeth more problems of root resorption are those with a periodontal support decreased, which may cause a degree of tooth mobility and bone loss alveolar. 4 degrees of severity according to the level of root resorption which is used to classify the severity of lesión3 are arranged:

i. Without reabsorption.

ii. Irregular contours.

iii. Presence of less than $3 \mathrm{~mm}$ reabsorption.

iv. Loss of $2 \mathrm{~mm}$ and a third of the LR.

v. Decreased root, more than one third radicular11 reabsorption.

\section{Pre-orthopedic treatment recommendations}

The low level of knowledge of the legal representatives of certain patient, makes us take into consideration the explanation of the treatment and its side effects such as root resorption, it is also important to publicize the informed consent. Obtaining medical records which provide patient data such as family history, current condition, general health, intra and extra oral inspection, drug delivery and habits among others. The most significant to prevent root resorption diagnosis is the radiographic study before treatment, to help us evaluate the state of the teeth in which problems such as found: periapical lesions, fractures, roots atypically, agenesis, previous root resorption, endodontic-tooth. Treatment is a bit less risky in young patients because it better tolerate occlusal changes, these data help prevent complications during treatment.

\section{Recommendations for orthopedic treatment}

There are several considerations to be taken into account during treatment as the implementation of hyperelastic devices, and application of light forces and intermittent. It should be borne in mind that pulp vitality and coloration did not present changes in cases of extensa. reabsorption orthodontic forces applied can modify the pulp bloodstream, rarely presenting necrosis of the pulp, for this must take into account that a tooth with vitality is reabsorbed more than a necrotic tooth or endodontic. Must be very careful and be aware of the central and lateral incisors as they are more susceptible to problems 
during application of force. To maintain proper monitoring during treatment and avoid problems of resorption should be diagnosed every six to nine or twelve months Radiographic help, in order to prevent or detect root resorption, if any teeth are at risk is carried out every 3 months. If during treatment a moderate or severe root resorption is observed should reconsider the treatment plan to prevent mobility or excesiva.1 reabsorption As an alternative should be considered temporary suspension of treatment to decrease the degree of reabsorption radicular. In other cases if the injury is severe splint des occlusion at the end of treatment were applied, and if the continuous lesion must take the alternative root canal. It should be borne in mind that in all cases at the end of treatment should be avoided premature contacts and traumas that endanger the integrity of dental roots.

\section{Food recommendations for the use of orthopedic devices}

i. During orthodontic or orthopedic treatment should avoid eating hard foods: candies, beans, toast, ice, bones, etc.

Also avoid fibrous foods such as meat (grilled, fried, deep fried, etc.) are best avoided during the first month of treatment until the patient fits the aparatología http://www.ortodonciaestetica.pe/ brackets/, Then it is possible to consume precut; instead you can eat fish, chicken, etc.

It is recommended to eat fruit (apples, pears, watermelon etc.), as long as they are chopped (salads) and avoid biting.

Avoid any kind of sticky food throughout treatment (nougat, tooffes, frunas, mashmelos, gum), as these are stuck in appliances and plate collectors become bacterial.

\section{Oral hygiene recommendations}

i. Hygiene during treatment is essential in the use of appliances and devices since they are retentive, which means they accumulate more food, consequently will create more prone to tooth decay, gum disease and high risks contributes to root resorption due to loss of periodontal tissue.

ii. Oral cleaning should be performed after every meal and the one that should last for at least three minutes, for better cleaning can be supported initially using a clock to control time, this will help create a good habit.

iii. Proper technique is that where the brush provided toothpaste, it is placed perpendicular to the tooth surface and a rotary movement is performed (small circles). In corrective orthodontic we must go tilting the brush to clean all tooth surfaces and is very important to the area between the bracket and gum, where many accumulates food.

iv. Also within corrective orthodontics and interceptive orthodontics using special brushes as the use of interproximal brushes are useful as these special brushes can be found in any chain of drugstores in different brands or also the use of an electric toothbrush can benefit cleaning, but a conventional toothbrush properly used with good technique is sufficient for hygiene suitable.

v. In addition, the supplement hygiene with the use of other accessories (mouthwashes, dental floss, etc.) will help us clean the areas between the arch and the tooth where no access brush, improving a high percentage of cleaning the mouth. The use of the mouthwash is essential during treatment, as it will enhance the brushing action, its mode of use is to make mouthwashes or "swish" undiluted for 30 seconds, 2 times a day; usually they come with a dosing cap $15 \mathrm{ml}$.

vi. Another device that may be helpful is the "water pick" which is an irrigator (spear pressurized water to clean these areas of difficult access).

\section{Orthopedic aftercare basic recommendations}

i. Requirement of a radiographic study, upon completion of treatment.

ii. Conduct a further review to be completed treatment

iii. Sigua motivate the patient with oral hygiene to keep your oral health.

iv. Refer the patient for the first month after finishing treatment avoid harsh and irritating foods.

v. Avoid biting or chewing hard objects especially in nervous patients seeking calm biting something.

vi. Cooperatively request the patient to go to dental appointments every 6 months for revisión.

\section{Recommendations to follow orthopedic treatment in parts with root resorption}

In most cases in which this root resorption after orthopedic treatment, remove the apparatus, this method is responsible for lesions initiate a repair process in several cases in which resorptions become severe causing harmful movements during mastication bring result in injuries may follow progress. In those teeth having a very severe root resorption, a thorough radiographic follow-up is recommended until root resorption and decrease evidence is not so noticeable. Importantly, the cement begins to repair naturally once end the active resorption processes for this must be removed from causante 13 agent. If after removing the main cause of root resorption, and resorptions continue, proceed to a dental devitalization, in which treatment relies on the application of calcium hydroxide in the three months to give a total substitution percha after a year with this maneuver ankylosis looking teeth, which can be beneficial for the affected parts having high mobility. The consequences of root resorption after orthodontic treatment pose no threat to long-term for the patient. But we must take into account that in case of combined effects such as root resorption and bone loss in the alveolar ridge could be the cause of sequelae not innocuous.

\section{Results}

In this paper a study focused on the knowledge of orthopedic treatments were performed: o made to the legal representatives of patients treated at the Clinic of the Catholic University of Cuenca during the period March-July 2018, the approach was based on a number of 50 tutors in charge of the patient who underwent a survey final results gave as a percentage change in each question (Figure $1-8)$. 


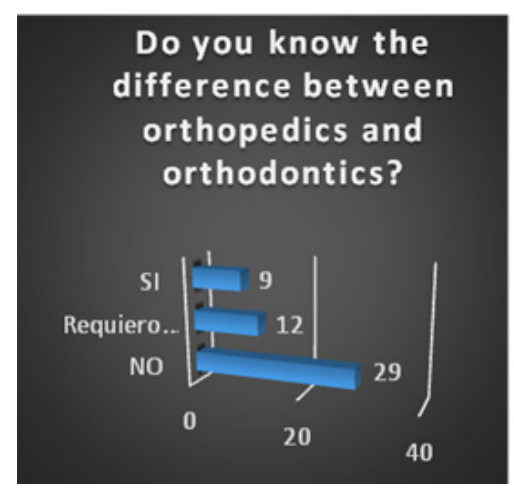

\section{Percent}

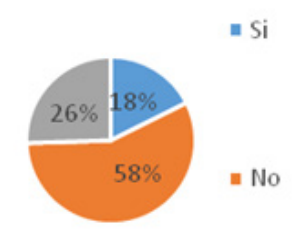

Figure I It discloses that most legal representatives do not know the difference between orthopedics and orthodontics represented 58\%, 26\% represents the partial knowledge about the subject and requires guidelines, while $18 \%$ have adequate knowledge due to past experiences.

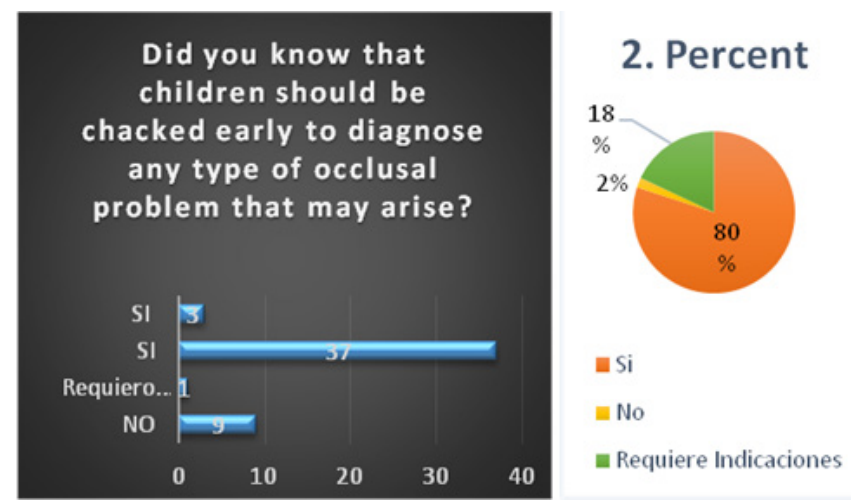

Figure 2 It shows that legal representatives have adequate knowledge about early diagnosis of malocclusions or developmental problems represented by $80 \%, 18 \%$ indicated that Reps in did not know that at an early age can diagnose any problems occlusal what help improve treatment.

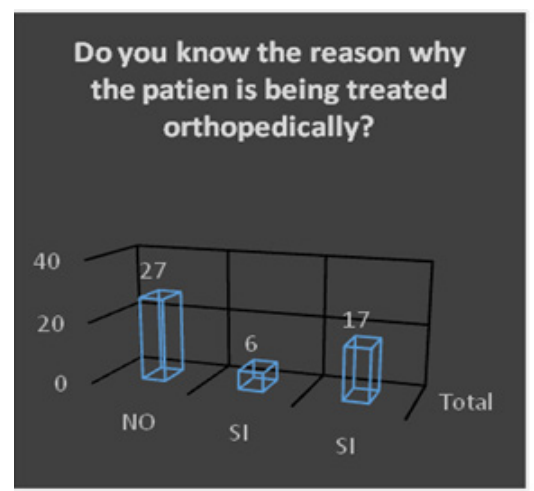

\section{Percent}

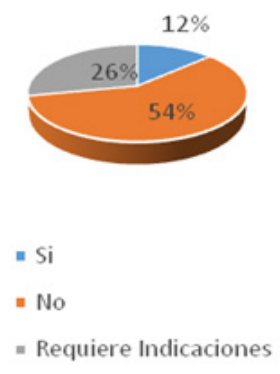

Figure $3 \mathrm{It}$ describes that most of the legal representatives do not know why the patient is being operated orthopedically with $54 \%, 26 \%$ corresponding to representatives requiring indications had a slight knowledge of the subject but could not express it so adequate and 12\% knew the cause for which the patient is being treated orthopedically, the most common reason dental crowding, giroversiones and lack of skeletal development.

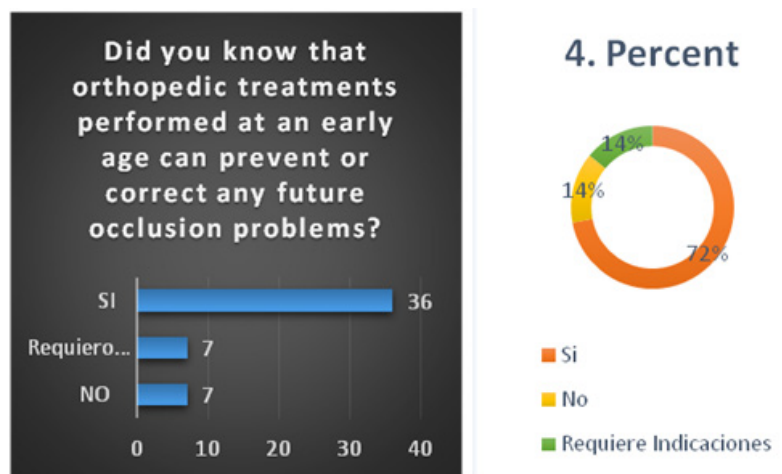

Figure 4 Determines that legal representatives acknowledge that the dental support is essential to prevent or correct problems in occlusion that are provided when they are performing at an early age represented $72 \%$ since they favor the treatment, $14 \%$ of representatives not they had knowledge about treatments orthopedically made when they are small they are easier to treat and less time commitment.
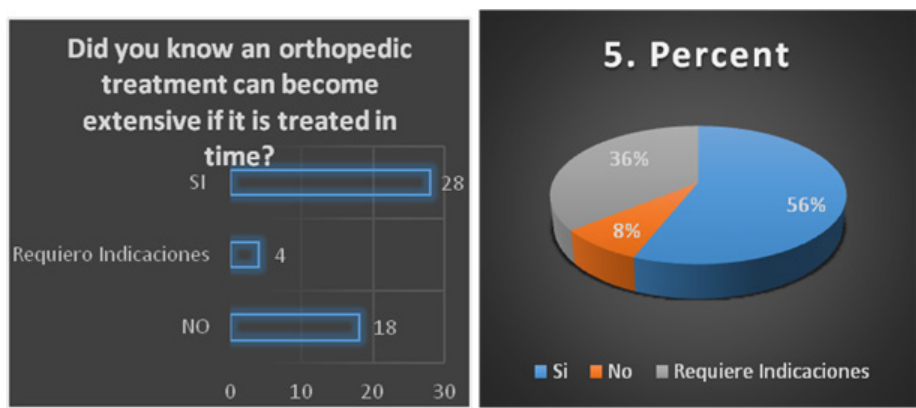

Figure 5 It suggests that $56 \%$ of legal representatives have adequate knowledge about that orthodontic treatment is prolonged when not diagnosed and treated at an early age, $36 \%$ and $4 \%$ were determined as one and having no knowledge about the topic required indications were mentioned and captured positively and favorably on mutual respect.

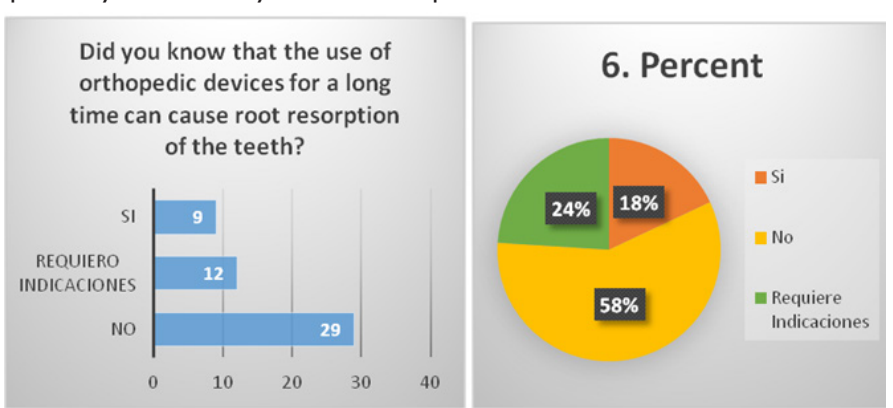

Figure 6 Describe that $58 \%$ of legal representatives do not have enough knowledge about a prolonged orthodontic treatment can cause root resorption and other more damaging consequences, $9 \%$ and $12 \%$ of guardians of these patients they had knowledge no so clear about the issue and their ideologies were due to bad past experiences that were consistent with the problems presented (root resorption, bone loss, tooth mobility, etc.) 


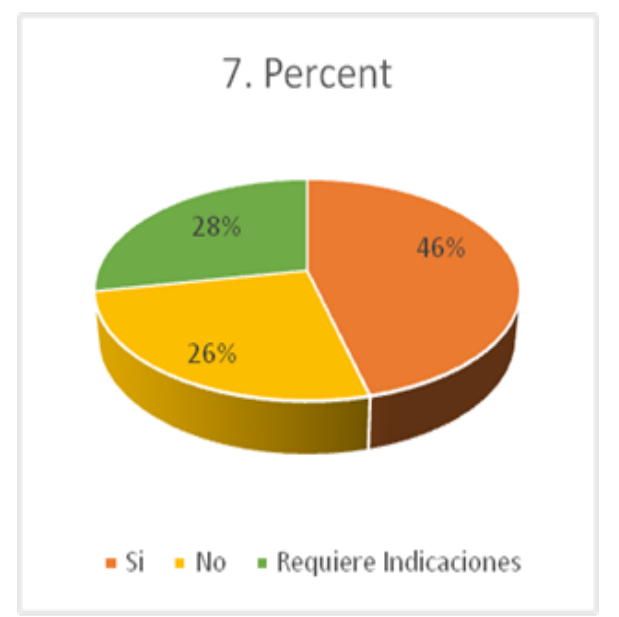

Figure 7 Discloses that $46 \%$ of legal representatives know that dental visits for patients who are being treated orthodontically are essential for exist thereof and visits are according to the progress of treatment, $28 \%$ mentioned that depends on schedules proposed by the professional but were instructed in a better way and $26 \%$ last had nothing base about dental appointments so that instruction to the representatives and patients was critical in this case.

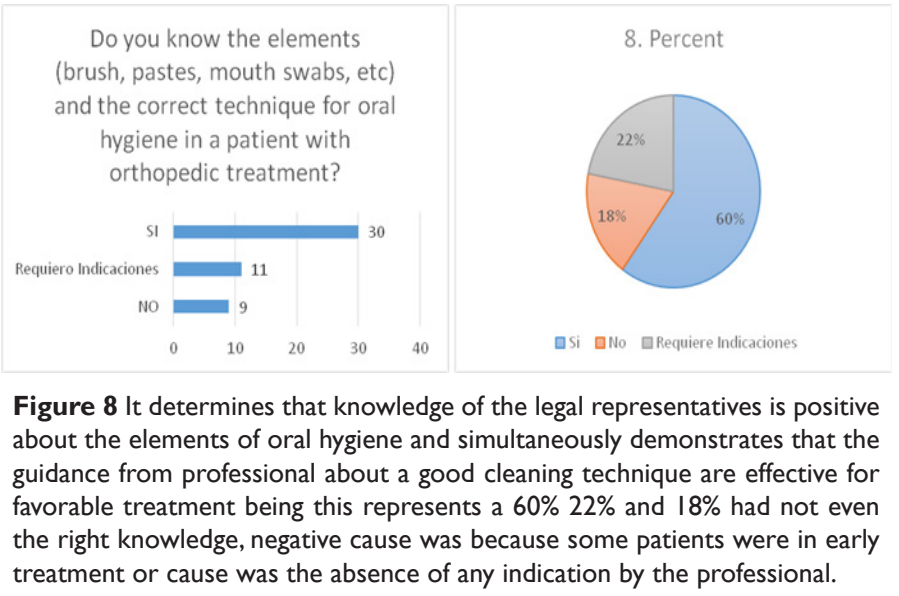

\section{Discussion}

This study determined on degree of knowledge of legal representatives in patients being treated orthopedically where recommendations mentioned before, during and after treatment in order to avoid problems such as root resorption and other unfavorable problems. Berrocal \& Col. determine that root resorption associated with orthodontic treatment with fixed appliances preventive apparatus or during the standard procedure, the radiographic monitoring every 6 months is necessary to know the outcome of treatment. However, Levander \& Col. mention that in his study in patients who have teeth with a high risk of root resorption and bone loss is recommended monitored every three months. Vaquero \& Col. mention that if resorption continues to progress will proceed to a compromised desvitalizar based structures, teeth with severe root resorption treatment, treatment vary in a splinting for its long term stability, which agrees with what Col. \& mention Chumi which stipulate that if the use of a splint severe injury is essential to avoid disclusion, which is what makes the end of treatment and if this continues to advance reabsorption is recommended based planning root canals for the affected parts. Chumi \& Col., Col. Berrocal \& Schwarz and agree that in several cases the root lesions initiate a repair process by removing the causative agent, so the withdraw or terminate the application of force is a way to avoid these preventable injuries because otherwise this can lead to increased root resorption and other feasible problems.

Bishara et al postulate that those parts having root injury and show cementum and dentin repaired added resistance to root resorption, which relates support to effect, followed by a three-month pause in treatment which stop root resorption. But according Vaquero these affected parts do not show an acceptable amount of cement and dentin repair to stop reabsorption. Varela \& Col. in their study suggest that in extreme cases of resorption and mobility is recommended to make the devitalization of the pieces, and then place calcium hydroxide for short periods of three months to replace completely by percha after a year, this is done in order that these pieces resorbed achieve a ankylosis for stabilization of same. To this Cowboy \& Col. they delimit that this application should be performed several preventive diagnostics with professional endodontist, in order to prevent severe resorption. All authors cited in this paper confirm that careful monitoring is essential after treatment ends, in order to avoid the existence of premature contacts, occlusal interference and nuisance which may further affect the integrity of the affected parts.

\section{Conclusion}

In conducting our research it was concluded that most parents do not know the difference orthopedics and orthodontics but equally take their children to orthopedic treatment because of the limited information they have is that it is removing the crowding tooth of the teeth without knowing much of the treatment. But they have positive knowledge of the elements of oral hygiene but do not know how to perform dental hygiene properly due to lack of information professionals traffickers. It could also conclude that most of the parents of these children know and are aware that a good dental and orthopedic treatment depends on frequent views dentist to correct their dental condition and give a good occlusion and aesthetics child patient. In the same way delve who do not know whether the treatment brings consequences for long stretches, but equally attend the faculty of the University because they want to prevent a crowded teeth future of their children and thus get dental harmony.

\section{Acknowledgements}

None.

\section{Conflict of interest}

The author declares no conflicto of interest.

\section{References}

1. Cristina Sánchez Escobar, Andrea V, Guerrero Villegas Dra. Juan Hector Ramirez Vladimir de Jesus Montes. root resorption in orthodontic retreatment - Literature Review. Review literatura. 2017;9(1-5).

2. Pamela Elizabeth Ruiz Reascos. apical external root resorption in incisors and upper and lower canines undergoing orthodontic treatment early stage.

3. Berrocal A. Root resorption induced orthodontic treatment. Costa Rica: ULACIT; 2011. $18 \mathrm{p}$.

4. Vaquero P, Perea B, Labajo E, et al. Root resorption during orthodontic treatment: causes and recommendations for action. Cient Dent. 2011;8(1):61-70. 